\title{
Backcalculation of Residual Tensile Strength of Regular and Ultra High Performance Fiber Reinforced Concrete From Flexural Tests
}

\author{
Barzin Mobasher ${ }^{1}$, Mehdi Bakhshi ${ }^{2}$, Christopher Barsby $^{3}$
}

\begin{abstract}
The tensile stress-strain response of a fiber reinforced concrete dominates the performance under many loading conditions and applications. To understand this response, a back-calculation process from flexural testing is employed to measure the material properties. The procedure is performed by model fitting of the experimental three-point and four-point bending load deflection data on two types of macro synthetic polymeric fibers, one type of steel fiber and one type of Alkali Resistant (AR) glass fiber. A strain softening tensile model is used to simulate the behavior of different FRC types and simulate the experimental flexural response. The stressstrain model for each age, fiber type and volume fraction is predicted by means of the inverse analysis procedure, using closed-form moment-curvature relationship. The method of approach is further applied to one external data set for Ultra High Performance Fiber Reinforced Concrete (UHPFRC) with two different types of steel fibers and validated by tensile test results reported. Results of back-calculation of stress-strain responses by tri-linear tensile model for all mixtures are compared and correlated with the corresponding standard method parameters used for post crack behavior characterization and a regression analysis for comparative evaluation of test data is presented.
\end{abstract}

\section{keywords}

Concrete, Fiber reinforced concrete, Flexural behavior, Moment-curvature response, Postcracking tensile strength, Stress-strain response.

\section{Introduction}

Fiber reinforced concrete is widely used in infrastructure applications because of improved mechanical properties such as fracture toughness, ductility, durability, and crack-width control $[1,2,3,4,5]$. Steel, glass, natural, and synthetic fibers have been used over 40 years in industrial slabs, floors, and pavements to primarily reduce shrinkage and thermal cracking [6, 7], reduce the required slab thickness, and increase the allowable joint spacing $[8,9,10,11,12,13,14]$. Experimental tests show that fibers increase the flexural and ultimate load carrying capacity in proportion to their volume and aspect ratio $[15,16,17,18,19,20]$. Figure 1 shows application of fiber reinforced concrete in elevated slabs and water distribution infrastructure. Structural

1 Corresponding Author, School of Sustainable Engineering and Built Environment, Arizona State University, Tempe, AZ 85287-5306. (P) (480)965-0141, (F) (480)965-0557, (E) barzin@ asu.edu 2 AECOM, New York, NY 10005

3 PK Associates Structural Engineers, Scottsdale, AZ, 85250 
applications of fibers include but are not limited to precast structural elements [21], tunnel linings [22, 23], shotcrete [24, 25, 26, 27, 28], offshore structures, seismic applications, thin and thick repairs, crash barriers, footings, and hydraulic structures [29, 30]. The fibers are also added to concrete to enhance spalling resistance during exposure to high temperature [31].

The mechanical properties depend on the characteristics of the concrete matrix but also on the type and geometry of the fibers that governs their bond mechanism with the matrix [32, 33]. Fibers offer increased abrasion and impact resistance as well [34]. The effectiveness of short, randomly distributed fibers may be superior to other forms of reinforcement such as welded wire mesh, or rebars since the small diameter of the individual fibers ensures a more uniform dispersion, along with a far superior bond strength. Moreover, due to the reduced specific spacing, fibers strengthen the composite at the micro level by bridging the microcracks before they reach the critical flaw size [35]. Among all mechanical parameters, residual tensile strength and toughness are the most improved parameters which are a direct consequence of fiber bridging mechanisms across the crack surfaces [36, 37].

Mechanical properties are often characterized by flexural tests, however the scatter and variations in testing such as notched or unnotched samples, or the choice of control variable used in the experimental results, are compounded by the various methods to report the experimental results of the post-peak region. For example, scatter is much smaller for synthetic fibers than steel fibers due to the higher number and their more homogeneous distribution across the fracture surface [36]. Scatter is also lower for samples such as round panel specimens tested under ASTM C1550 than ASTM C1609 [38] specimens. Scatter in the case of ASTM C1609 may also be attributed to the degree of rigidity of the support reactions, or frictional sliding at the supports.

Several series of flexural test results from different experiments are combined with in a procedure to backcalculate tensile properties from flexural results. The test results are also evaluated using available standard test methods such as ASTM C1609 [38], RILEM TC 162TDF [39], and JCI-SF4 [40] which propose calculation of residual strength using simple engineering bending theory for linear elastic materials and uncracked section properties. The equivalent tensile properties are compared with the residual strength measures obtained from these standards and compared based on the effects of age, fiber type, length, and sample size on flexural load-deflection.

\section{Flexural Test}

This paper validates a back-calculation procedure for flexural test results and obtains tension stress-strain response from a variety of tests conducted on notched and un-notched beams of different sizes, fiber types, shapes, lengths, and volume fractions. The objective is to correlate the empirical residual strength methods of Rilem, and ASTM and JCI approaches using the proposed back-calculation approach that is based on a closed-form solution based on moment curvature relationship.

A database containing three internal and one external data set was used for analysis. All internal experiments were conducted at the Structural Engineering Laboratory at the Arizona State University. Three data sets consisted of Set 1 with two Polymeric fibers of modified 
Polypropylene, Polyethylene and Olefin blends, both at volume fraction of $3 \mathrm{~kg} / \mathrm{m}^{3}\left(5 \mathrm{lb} / \mathrm{yd}^{3}\right)$. Set two consisted of one type of Steel fibers at three different volume fractions, and Set 3 consisted of AR Glass fibers at three different fiber lengths. All samples were tested under flexural testing configuration and the load-deformation response in the post-peak region was measured. Physical and mechanical properties of the fibers used in the test program are presented in Table 1.

The analysis section also discusses results from published work on four different mixtures of High Performance Fiber Reinforced Concrete (HPFRC) by Kim et al. [41]. This was designated as Set 4 and included both tensile and flexural test results.

\subsection{Testing Program}

Proportions of eight different mixtures prepared and tested under three-point bending configuration are shown in the Table 2. The first letter on the samples' labels refers to the general type of fiber used, i.e. " $\mathrm{P}$ " in case of polymeric, " $\mathrm{G}$ " in case of glass fiber and " $\mathrm{S}$ " in case of steel fiber. The following number is the dosage of the fiber presented in $\mathrm{kg} / \mathrm{m}^{3}$. For polymeric and steel fibers, the letter following this number refers to the type of fibers shown in Table 1, while for glass fibers, the number following this number is the length of fiber. A final number at the end of the labels designated the age at testing. In addition to the samples tested, one set of published UHPFRC data by Kim et al. [41] was used with employed two different types of steel fibers, "H" for hooked fibers and designation " $T$ " was introduced to refer to longitudinally twisted steel fibers. Subsequently, parameter "L" refers to large size of specimen with depth, width and span of 150,150 and $450 \mathrm{~mm}$, respectively, to differentiate the results from results of medium size specimens reported by Kim et al. [41].

Closed loop control flexural tests were conducted on pre-notched FRC samples of polymeric and AR glass in accordance with RILEM TC 162-TDF recommendation in order to monitor postpeak response [39]. Dimensions of Set 1 Polymeric-FRC sample and Set 2 AR glass-FRC samples were $450 \mathrm{~mm} \times 100 \mathrm{~mm}$ x $100 \mathrm{~mm}$ with an initial notch length of $12 \mathrm{~mm}$ and test span of $400 \mathrm{~mm}$. Unnotched steel-FRC samples in Set 3 were tested in accordance with ASTM C1609 under four-point bending loading configuration using $450 \mathrm{~mm}$ x $150 \mathrm{~mm}$ x $150 \mathrm{~mm}$ specimens. The diameter of steel fibers used was $0.3 \mathrm{~mm}$.

Tests were performed under closed loop control with Crack Mouth Opening Deformation (CMOD) or load point deflection as the controlled variable. Both the CMOD and deflection were measured using a Linear Variable Differential Transformer (LVDT) with a working range of 2.5 $\mathrm{mm}$. Cracks initiated from the notch and extended up along the depth of the beam. The crack opening was resisted by bridging fibers which pulled out under this loading. The presence of fiber significantly increases the ductility and resulted in a stable crack opening up to high range of deflections. The load-deflection curve is characterized by the maximum load and its associated deflection, elastic stiffness, maximum flexural strength and flexural toughness. The post-peak behavior of the samples was also reported as elastically equivalent residual strengths as measured by three alternative methods of ASTM C1609 $\left(f_{150}^{D}\right)$, JCI-SF4 $\left(\sigma_{b}\right)$ and RILEM TC 162-TDF $\left(f_{e q, 3}\right)$. 


\subsection{Effect of Fiber Type on Strength and Flexural Toughness}

Results of experimental analysis on three- and four-point bending tests on different macro synthetic, glass and steel fibers are summarized in Table 3. A wide range of responses in the data such as apparent flexural strength and toughness correlate with the fiber type, volume fraction, loading rate, and age at testing. The toughness measure is obtained as the area under the load deflection curve and as an age-dependent property correlates with strength gain, therefore toughness after 28 days of curing was used as the control and results obtained after 14 and 56 days for steel fibers were correlated with the 28 day results.

Effect of curing duration on flexural response of polymeric fiber types A and B in Set 1 are shown in Fig. 3a. Average elastic flexural stiffness of P3-A samples increased by 30\%, both deflection and load associated with the maximum load level increased by 11 and $17 \%$ respectively, and toughness doubled from 14 to 28 days. The increase in apparent flexural strength from 14 to 28 days was from 1.57 to $1.92 \mathrm{MPa}(+22 \%)$. In P3-B samples with $3 \mathrm{~kg} / \mathrm{m}^{3}$ of type B polymeric fibers, no significant change in elastic flexural stiffness was observed. However, the maximum load and its associated deflection increased by 18 and $17 \%$ respectively, while the toughness increased from 2.2 to $4.3 \mathrm{kN}$.mm (+95\%) from 14 to 28 days.

It is clear that the primary parameter that differentiates among the age of these systems is the toughness which is affected by the post cracking response. When overall toughness is specified as a design parameter, standard procedures can be utilized to select fiber type, length, and volume content. Such procedures, however, are costly and vary for each fiber type. It would be ideal to develop a procedure to back-calculate the tensile response from each flexural test so that the design procedures can utilize these results.

Effect of fiber length at 28 days on flexural response of AR-glass fibers in Set 2 are shown in Fig. $3 \mathrm{~b}$ for three different fiber lengths of 6,12 and $24 \mathrm{~mm}$. Results show that the glass fiber length does not affect the deflections at maximum flexural load; however, flexural strength increased by $12 \%$ from 5.79 to $6.50 \mathrm{MPa}$ at 28 days as the fiber length changes from 12 to 25 $\mathrm{mm}$. The flexural toughness is showing a marginal decrease with increasing fiber length.

Comparative evaluation of the mixtures shows that there is no discerning of the effect of fiber type at these loading levels, and as shown in Figure 3, minimal changes are observed for all mixes of Set 1, namely P3-A, P3-B, and Set 2 Glass fiber mixtures. As far as flexural strength is concerned, little or no effect on the effect of age or fiber length is observed. The flexural ductility, however, is clearly affected in both cases of polymeric and glass fibers. The general increase in the post peak response from 14 to 28 days is therefore the main parameter affected by the curing duration.

Effect of steel fiber volume fraction and type are shown in Figure 4 of sample Set 3. Note that for the low volume contents in the range of 13 to $26 \mathrm{~kg} / \mathrm{m}^{3}$, the effect of steel fibers is observed in the post crack response while as the fiber content increases, behavior changes from strain softening to strain hardening. The transition from strain softening to hardening is best shown by the increase in the ultimate strength and post crack resistance in terms of toughness. 


\section{Strain Softening and Hardening Models}

A formulation is presented to back-calculate material properties by fitting experimental data with a closed form relationship of the load deflection using a nonlinear material model $[42,43,44]$. The adaptation of this tri-linear model provides a precise correlation of the flexural response to back-calculate material parameters and could explain the differences between the tensile and flexural strengths of strain hardening and strain softening composites [45, 46].

Fig. 5 presents the constitutive model for homogenized strain softening/hardening fiber reinforced concrete. The tension model in Fig. 5a is described by a tri-linear response with an elastic range $E$, first cracking tensile strain $\left(\varepsilon_{c r}\right)$ and post cracking modulus $E_{c r}=\eta E$, which $\eta$ is assigned a negative or positive scalar value in order to simulate either strain softening or hardening materials. The third region in the tensile response is a constant stress range defined with stress $\sigma_{c s t}$ in the post crack region. Two strain measures define the first cracking and transition strains $\left(\varepsilon_{c r}, \varepsilon_{t r n}\right)$. The tensile response terminates at the ultimate tensile strain level of $\varepsilon_{t u}$. The linear portion of an elastic-perfectly-plastic compressive stress-strain response terminates at yield point $\left(\varepsilon_{c y}, \sigma_{c y}\right)$. The response remains constant at compressive yield stress $\sigma_{c y}$ until the ultimate compressive strain $\varepsilon_{c u}$ as shown in Fig. 5b. To convert this approach for closedform solution of moment-curvature response and load deflection calculation, parameters must be expressed in normalized terms. Two intrinsic material parameters of first cracking tensile strain $\varepsilon_{c r}$ and tensile modulus $E$ are used to define seven normalized parameters as shown in Figures 5a and $5 \mathrm{~b}$ and Eqs. (1):

$$
\omega=\frac{\varepsilon_{c y}}{\varepsilon_{c r}} ; \quad \alpha=\frac{\varepsilon_{t r n}}{\varepsilon_{c r}} ; \quad \beta_{t u}=\frac{\varepsilon_{t u}}{\varepsilon_{c r}} ; \quad \lambda_{c u}=\frac{\varepsilon_{c u}}{\varepsilon_{c r}} ; \quad \gamma=\frac{E_{c}}{E} ; \quad \eta=\frac{E_{c r}}{E} ; \quad \mu=\frac{\sigma_{c s t}}{E \varepsilon_{c r}}
$$

In a flexural test the derivation of moment curvature diagram for a rectangular cross section with $a$ width $b$ and depth $d$, the Kirchhoff hypothesis is applied and the maximum tensile strain $\beta$ and maximum compressive strain $\lambda$ are linearly related through the normalized neutral axis parameter, k. as in Eqs. (2)

$\beta=\frac{\varepsilon_{t b o t}}{\varepsilon_{c r}} ; \quad \lambda=\frac{\varepsilon_{c t o p}}{\varepsilon_{c r}} ; \quad \frac{\lambda \varepsilon_{c r}}{k d}=\frac{\beta \varepsilon_{c r}}{d-k d} \quad$ or $\quad \lambda=\frac{k}{1-k} \beta$

Using the normalized parameters defined in Eqs. (1) and (2), stress strain responses and toughness $\mathrm{G}_{\mathrm{f}}$ are expressed as:

$$
\begin{aligned}
& \frac{\sigma_{c}(\lambda)}{E \varepsilon_{c r}}=\left\{\begin{array}{ll}
\gamma \lambda & 0 \leq \lambda \leq \omega \\
\gamma \omega & \omega<\lambda \leq \lambda_{c u} \\
0 & \lambda_{c u}<\lambda
\end{array} \quad \frac{\sigma_{t}(\beta)}{E \varepsilon_{c r}}= \begin{cases}\beta & 0 \leq \beta \leq 1 \\
1+\eta(\beta-1) & 1<\beta \leq \alpha \\
\mu & \alpha<\beta \leq \beta_{t u} \\
0 & \beta_{t u} \leq \beta\end{cases} \right. \\
& G_{f}=\frac{1}{2} E \varepsilon_{c r}^{2}\left[\alpha-\mu\left(1+\alpha-2 \beta_{t u}\right)\right]
\end{aligned}
$$


By assuming linear strain distribution across the depth and ignoring shear deformations, stress distribution across the cross section at three stages of imposed tensile strain: $0 \leq \beta \leq 1,1<\beta \leq \alpha$ and $\alpha<\beta \leq \beta_{t u}$ are obtained in closed form [45]. Internal moment is obtained using the force components and their distance from the neutral axis and the curvature is determined as the ratio of compressive strain at top fiber $\left(\varepsilon_{c t o p}=\lambda \varepsilon_{c r}\right)$ to the depth of neutral axis $k d$. The moment $M_{i}$ and curvature $\phi_{i}$ at each stage $i$ are then normalized with respect to the values at cracking $M_{\mathrm{cr}}$ and $\phi_{\mathrm{cr}}$ and presented in Eqs. (5) and (6). The transition from deflection softening to deflection hardening is defined by critical normalized post-peak tensile strength $\left(\mu_{\text {crit }}\right)$ as defined in Eq. (7).

$$
\begin{aligned}
& M_{i}=M^{\prime} M_{c r} ; \quad M_{c r}=\frac{1}{6} b d^{2} E \varepsilon_{c r} \\
& \phi_{i}=\phi_{i}{ }^{\prime} \phi_{c r} ; \quad \phi_{c r}=\frac{2 \varepsilon_{c r}}{d} \\
& \mu_{c r i t}=\frac{\omega}{3 \omega-1}
\end{aligned}
$$

Calculation of $k, M^{\prime}$ and $\phi^{\prime}$ for the five stages of governing strain is presented in Table 4 and also shown in Figure 6. During stage 1 the tensile and compressive zones are both elastic with a linear moment-curvature plot and the neutral axis at the centroid of the sample. This case continues until the point of first cracking. There are two potential regions when the elastic Stage 1 ends and the tensile cracking as defined in Stage 2 starts. The compression side may or may not enter the plastic zone. Elastic compression is denoted as Stage 2.1, while tensile cracking, with the compression in plastic range is defined as Stage 2.2 (tension-plastic compression).

Two potential regions at the end of Stage 2 depending on whether the transition takes place form region 2.1 or 2.2 exist. Stage 3.1 is an elastic response in compression while plastic compression is defined as Stage 3.2. It is important to note that depending on the relationship among material parameters, any of the stages 2.1 , and 2.2, or 3.1, and 3.2 are potentially possible in succession.

By applying the moment-area method to the bilinear moment curvature response, mid-span deflection of three-point bending tests can be derived explicitly [42]. After cracking, the curvature distribution depends on the normalized post-peak tensile strain. The maximum deflection during the elastic stage of loading is determined from the curvature at cracking $\left(\phi_{\mathrm{cr}}\right)$ and Eq. (8). If $\mu>\mu_{\text {crit }}$, as the post-crack curvature increases, the moment continues to increase with the deflection determined by Eq. (9). On the other hand, if $\mu<\mu_{\text {crit }}$, as the post-crack curvature increases, the moment either increases or decreases at the levels below the bilinear cracking moment $M_{\mathrm{cr}}$, the deflection during this stage is determined by Eq. (10), and the term $L_{p}$ represents the length of localization zone.

$$
\begin{aligned}
& \delta_{c r}=\frac{1}{12} L^{2} \phi_{c r} \\
& \delta_{u}=\frac{L^{2}}{24 M_{u}^{2}}\left[\left(2 M_{u}^{2}-M_{u} M_{c r}-M_{c r}^{2}\right) \phi_{u}+\left(M_{u}^{2}+M_{u} M_{c r}\right) \phi_{c r}\right] \\
& \delta_{u}=\frac{\phi_{u} L_{p}}{8}\left(2 L-L_{p}\right)+\frac{M_{u} \phi_{c r} L}{12 M_{c r}}\left(L-2 L_{p}\right)
\end{aligned}
$$


From the approximate bilinear moment-curvature diagram, the total load $P_{i}$ at a given stage of loading $i$ can be calculated by Eq. (11) for $\phi_{1}$ through $\phi_{\mathrm{u}}$, where $S=L / 2$ for three point bending tests, respectively.

$$
P_{i}=\frac{2 M_{i}}{S}
$$

When a flexural specimen is loaded well into the post peak region, two distinct zones develop and the deformation localizes in the cracking region, while the remainder of the specimen undergoes general unloading. To correlate the stress-crack width relationship into the stressstrain approach, localization is treated as an average response within the cracking region. Results are used as a smeared crack in conjunction with the moment-curvature diagram to obtain load deformation behavior as presented by Soranakom and Mobasher [45][47]. By changing the crack localization length, $L_{p}$, the presented model responds by a general softening of the post peak zone in the load deflection curve and shown by Fig. 7. The length of localization only affects the descending portion of the response. It is important to note that the simulated residual load capacity is not sensitive to the crack localization length at deflections in excess of $0.5 \mathrm{~mm}$.

\section{Analysis - Prediction of Load-Deflection Response of FRC}

The back-calculation procedure computes the tensile material properties from experimental three- and four-point bending load-deflection data. Results of back-calculation of stress-strain responses by trilinear tensile model for all mixtures are shown in Table 5. Figures $7 \mathrm{a}$ and $\mathrm{b}$ represent the effect of curing time on the back calculated tensile stress-stain response and flexural load-deflection response of type A and B macro synthetic fibers. The initial response is linear elastic up to the first crack stage at about $2 \mathrm{MPa}$ for 14 day and increased to 2.3-2.6 MPa for 28 day samples. After cracking, load is transferred to the fibers bridging the cracks resulting in the significant drop in the sample stiffness and increasing the crack width. Back-calculated tensile stress-strain responses show that after an average strain level of about 0.003-0.004 $\mathrm{mm} / \mathrm{mm}$, the residual strength of the macro synthetic fiber composites reaches a constant value and that strain is maintained until 3-4\% level. The post-crack residual strength at this plateau zone increased from about 0.4 to $0.7 \mathrm{MPa}$ between 14 to 28 days.

The load versus deflection response based on the simulated fit of the data matches the experimental response as shown in Figure $7 \mathrm{~b}$. The overall predictions are well established. Representative properties for the simulation of upper and lower bound values obtained from these samples indicate $\mathrm{E}=18-21 \mathrm{GPa}, \alpha=30-40, \mu=0.21-0.3, \eta=0.02-0.026$ and $\varepsilon_{c r}=107-125$ $\mu$ str. In all these fits, the parameters for the ratio of compressive to tensile stiffness and strength were held constants at $\gamma=1$, and $\omega=10$. The limits of the modeling were set at $\beta_{t u}=267-406$ and $\lambda_{c u}=70$.

Back-calculated tensile stress-strain response and experimental and simulated load-deflection response for glass fibers are shown in Figs. 8a and b, respectively. The tensile strength of the glass fiber campsites are affected only marginally by the fiber length as the tensile strength increased from about 2.92 to $3.6 \mathrm{MPa}$ by increasing the fiber length from 6 to $24 \mathrm{~mm}$. The back- 
calculated tensile strength for parameter $\mu$ in this case is $0.11,0.05$ and 0.06 , representing the effect of fiber length from 6 to 12 and $24 \mathrm{~mm}$ and corresponds to residual tensile strength at the plateau zone for glass fiber reinforced samples in the range of 0.2 to $0.3 \mathrm{MPa}$. Representative properties for the simulation of upper and lower bound values obtained from these samples indicate $\mathrm{E}=32-33 \mathrm{GPa}, \alpha=20-42, \mu=0.06-0.11, \eta=0.023-0.049, \varepsilon_{c r}=90-110 \mu$ str. The simulated load-deflection responses show good agreements with experimental data and the descending part of load-deflection response is fitted quite well.

Effect of steel fiber was evaluated using different dosages of 13, 26 and $39 \mathrm{~kg} / \mathrm{m}^{3}$ using data from Set 3. Hooked-end steel fibers designated as type $\mathrm{H}$ fiber were used in the concrete mixes poured into samples specified as type L specimens $(450 \mathrm{~mm} \times 150 \mathrm{~mm} \times 150 \mathrm{~mm})$. At 28 days, the steel fiber reinforced samples showed increases in flexural toughness as fiber dosage increased. This is evidenced by the calculation of the area under load-deflection diagram shown in Fig 9b. Flexural toughness increased by $43 \%$ and $165 \%$ by increasing steel fiber dosages from $13 \mathrm{~kg} / \mathrm{m}^{3}$ to $26 \mathrm{~kg} / \mathrm{m}^{3}$ and $39 \mathrm{~kg} / \mathrm{m}^{3}$, respectively. The residual flexural loads increased proportionally with the fiber dosage. While this improvement is clearly evident in the measured toughness $\left(\mu=0.12-0.15\right.$ to $0.22-0.33$ and to 0.42 for the 13,26 and $39 \mathrm{~kg} / \mathrm{m}^{3}$ dosages, respectively), first crack tensile strength is not largely affected by the fiber dosage and is stable at around 1.89, 1.95 and 1.84 MPa with increasing fiber content. Simulations presented in Fig. $9 \mathrm{~b}$ are reasonable fits for the 13 and $26 \mathrm{~kg} / \mathrm{m}^{3}$ dosage curves, but fail to capture the almost linear unloading in the post cracking region of the $39 \mathrm{~kg} / \mathrm{m}^{3}$. This may be attributed to uneven distribution of fibers in the mix or larger concentrations of steel in the tensile region. Representative properties for the simulation of upper and lower bound values obtained from these samples indicate $\mathrm{E}=21-31 \mathrm{GPa}, \alpha=8-13, \eta=0.049-0.126, \varepsilon_{c r}=54-89 \mu$ str. The choice of the model used in the back calculation procedure may be altered using parameters, $\alpha$ and , $\eta$ to change from a strain softening to strain hardening model to properly capture both the peak and residual strength values. It is noted that there is a clear and consistent post crack residual strength measure that is similar to metal plasticity as the yielding behavior extends to deflections in excess of $4 \mathrm{~mm}$.

\section{Model Extension to UHPFRC}

Kim et al. [41] performed an experimental study on the effect of Hooked (H) and twisted (T) steel fibers on flexural and tensile responses of high strength cementitious matrix (84MPa) with a fiber content of $79 \mathrm{~kg} / \mathrm{m}^{3}$. Flexural tests were performed on three different geometries of specimens, $\mathrm{S}$ (small) for $50 \mathrm{~mm} \times 25 \mathrm{~mm} \times 300 \mathrm{~mm}$ specimens, $\mathrm{M}$ (medium) for $100 \mathrm{~mm} \times 100$ $\mathrm{mm} \times 300 \mathrm{~mm}$ and L (large) for $150 \mathrm{~mm} \times 150 \mathrm{~mm} \times 450 \mathrm{~mm}$. Properties of hooked fibers in this study are very similar to the ones presented in the previous section, with the exception of length of fibers and diameter of the hooked fibers which are $30 \mathrm{~mm}$, and $0.38 \mathrm{~mm}$ in Kim's study, respectively. The ratio of water to cementitious materials was 0.26 , and other details of mix design can be found in the reference paper [41].

Present method of approach is validated by comparing results of back calculated stress-strain responses with experimental tensile results. As shown in Fig 10, the present approach predicts the experimental results quite well. The results of flexural tests on HPFRC are also shown in Figure $11 \mathrm{~b}$ which represents the comparison of two steel fiber types of hooked $(\mathrm{H})$ and twisted (T) at two different specimen sizes. The fiber content in all mixtures is $79 \mathrm{~kg} / \mathrm{m}^{3}$. The UHPFRC 
showed very clear delineations between sample size ( $\mathrm{M}$ or $\mathrm{L})$ and fiber deformation type $(\mathrm{H}$ or $\mathrm{T})$. The twisted fibers in both the $\mathrm{M}$ and $\mathrm{L}$ sample sizes showed increases in flexural toughness, $(+116 \%)$ and hooked fibers $(+190 \%)$.

Peak tensile strength of about $5 \mathrm{MPa}$ and peak flexural strength in the range of 12-14 MPa are observed in these samples and do not seem to be influenced by sample size and fiber distributions. The maximum loads are 40,57, 87 and $98 \mathrm{kN}$ for the HM, TM, HL and TL samples respectively. The larger (L) samples show a slightly higher deflection capacity with $6 \mathrm{~mm}$ total deflection compared to the medium (M) samples at $4 \mathrm{~mm}$ deflection. This additional ductility could be from the combined effect of length and high volume fraction of steel fibers which deform and yield as the load increases.

Back-calculated tensile stress-strain responses resulted in simulated load-deflection responses for UHPFRC mixtures with steel fibers are shown in Fig. 11a and b for the Twisted fibers with the flexural simulation which compare the twisted and hooked fibers and show an excellent fit for the experimental data through sample failure. Parameters related to this simulation are summarized in Table 6. As shown in this table and Fig. 11a, back calculated stress-strain responses for hooked fibers for both medium and large size specimens are very similar. Back calculated stress-strain responses for twisted fibers however differ from medium to large size samples. The first crack tensile strength of the twisted fibers in medium specimens are $30 \%$ higher than large specimens which may be attributed to a more uniform fiber distribution in large samples. The back-calculated tensile strength parameter $\mu$ in cases of both fiber are also as much as $35 \%$ higher for medium size specimens. Similar to residual stress parameter, transitional tensile strains are $20+25 \%$ more in favor of medium size samples, but ultimate tensile strains are almost identical for all samples. Nonetheless, the difference between flexural test results of different sizes are much more significant than predicted stress-strain responses using this method of approach.

\section{Residual Strength Comparison with ASTM C1609 $\left(f_{150}^{D}\right)$, RILEM, and JCI-SF4 $\left(\sigma_{b}\right)$}

Flexural FRC beams results are also analyzed using the data reduction approach according to ASTM C1609 [38]. Load and net deflection are recorded up to an end-point deflection of L/150. Residual strength $\left(f_{150}^{D}\right)$ is calculated using an elastically equivalent approach:

$$
f_{150}^{D}=\frac{P_{150}^{D} L}{b d^{2}}
$$

where, $L$ is the span length, $P_{150}^{D}$ is the residual load at net deflection of $L / 150, b$ and $d$ are the average width and depth. ASTM C 1609 method uses an elastically equivalent elastic measure and overestimates the residual uniaxial tensile strength $\mu E \varepsilon_{c r}$ obtained based on the present approach by almost three times. Therefore, it is imperative to note that the $f_{150}^{D}$ parameter is not even an equivalently elastic stress and can not to be associated with the post crack tensile strength parameter $\sigma_{c s t}$ in Fig. $5 \mathrm{~b}$. 
Similar to ASTM C 1609, JCI-SF recommends testing fiber reinforced concrete by third-point loading and measuring the net deflection by Linear Variable Differential Transformers (LVDTs). Equivalent flexural strength $\left(\sigma_{b}\right)$ is calculated by Eq. (13) [40].

$\sigma_{b}=\frac{T_{b}}{\delta_{t b}} \cdot \frac{L}{b d^{2}}$

where, $\sigma_{b}$ is the equivalent flexural strength $\left(\mathrm{N} / \mathrm{mm}^{2}\right), T_{b}$ is the flexural toughness (N.mm), $L$ is the span length $(\mathrm{mm}), \delta_{t b}$ is the deflection of $1 / 150$ of span $(\mathrm{mm}), b$ is the width of failed crosssection (mm) and $d$ is the height of failed cross-section (mm).

According to RILEM TC 162-TDF [39] bending test method can be used for the determination of residual flexural tensile strength as well. The tensile behavior is obtained by the loaddeflection curve of a simply supported notched beam of $150 \times 150 \mathrm{~mm}$ cross section and 500 $\mathrm{mm}$ loaded under three-point bending arrangement tested using CMOD (Crack Mouth Opening Displacement) control. The residual flexural tensile strength $\left(f_{e q, 3}\right)$ is defined with respect to $\delta_{3}$, defined as:

$\delta_{3}=\delta_{L}+2.65 \mathrm{~mm}(\mathrm{~mm})$

where, $\delta_{L}$ is the deflection at the limit of proportionality $(\mathrm{mm})$.

The energy absorption capacity, $D_{B z, 3}$ is measured as the area under the load-deflection curve up to a deflection $\delta_{3}$ and consists of two parts. The part that includes the influence of steel fibers $\left(D_{B Z, 3}^{f}\right)$ is used for calculation of the equivalent flexural tensile strength, $\mathrm{f}_{\mathrm{eq}, 3}$, by means of the following equation.

$$
f_{e q, 3}=\frac{3}{2}\left(\frac{D_{B Z, 3}^{f}}{2.5}\right) \cdot \frac{L}{b{h_{s p}}^{2}}
$$

where, $L$ is the span length $(\mathrm{mm}), b$ is the width of the specimen $(\mathrm{mm})$, and $h_{s p}$ is the distance between tip of the notch and top of cross section (mm).

As shown in Fig. 12, direct correlation of JCI residual strength and the present method indicate JCI-SF4 method overestimates the residual uniaxial tensile strength $\mu E \varepsilon_{c r}$ by as much as three times. The exact correction factor for the JCA method is $1 /(3.22)$. A plot of corresponding values from two tests reflects the relationship between the two residual strength measures. It is imperative to note that the $f_{150}^{D}$ parameter can be used as a tensile stress measure associated with the post crack tensile strength parameter $\sigma_{c s t}$ in Fig. 2, so long as this parameter is corrected by a scale factor of 1/(2.94). Correction factors for presented standard parameters are as follows:

$$
f_{150}^{D}=2.94 \mu \sigma_{c r}
$$


$f_{e q, 3}=3.10 \mu \sigma_{c r}$

$\sigma_{b}=3.22 \mu \sigma_{c r}$

Similar to other test methods, direct correlation of RILEM residual strength and the present method indicates that RILEM method overestimates the residual uniaxial tensile strength $\mu E \varepsilon_{c r}$ by as much as three times. Alternatively, standard residual flexural strength parameters can be correlated to the tensile strength by a coefficient factor of $1 / 3$. This value is in accordance with the draft of ACI 544.3R report based on the stress coefficients values adopted by Barros 2004 [48] who presented a linear relationship between tensile stress at large strains and flexural strength using a coefficient factor of 0.27 .

$\sigma_{3}=0.27 f_{R_{-} 4}$

It is noted that in the proposed methods for design by FIB [49], a correction factor of $1 / 3$ is used for scaling the parameter $f_{R 3}$ from flexural tests to obtain $f_{F t u}$ as the ultimate residual strength. This correction factor can be justified by calibration of various specimen sizes, and various fiber types and dosages. The proposed value and the present calculation therefore correlate quite well. The present approach can be used as theoretical justification for the empirical values obtained and used in the FIB model code.

Since the inherent assumption of the available standard method assumes that the neutral axis is still at the centroid of the specimen, and the stress distribution is linear throughout. This leads to very high nominal flexural stress levels in tension fiber which are far more than tensile strength. Extreme caution must be exercised in application of the ASTM 1609, JCI-SF4 and RILEM TC 162-TDF methods in design and analysis of fiber reinforced concrete sections, as the results show overestimation of the residual parameter by as much as 2.94-3.22 times.

\section{Conclusions}

Characterization of tensile-flexural strain softening of fiber cement composites with alternative fiber types, volume fractions and lengths shows that the presence of fiber significantly increases the ductility of the material. By applying the load deflection back-calculation technique one can generate tensile constitutive data with a higher degree of accuracy than the current standard methods. Using a closed form set of governing parameters and variables applied through each stage of material response, the stress distribution that considers a shifting neutral axis also provide a more accurate representation of the residual strength and toughness of FRC. 


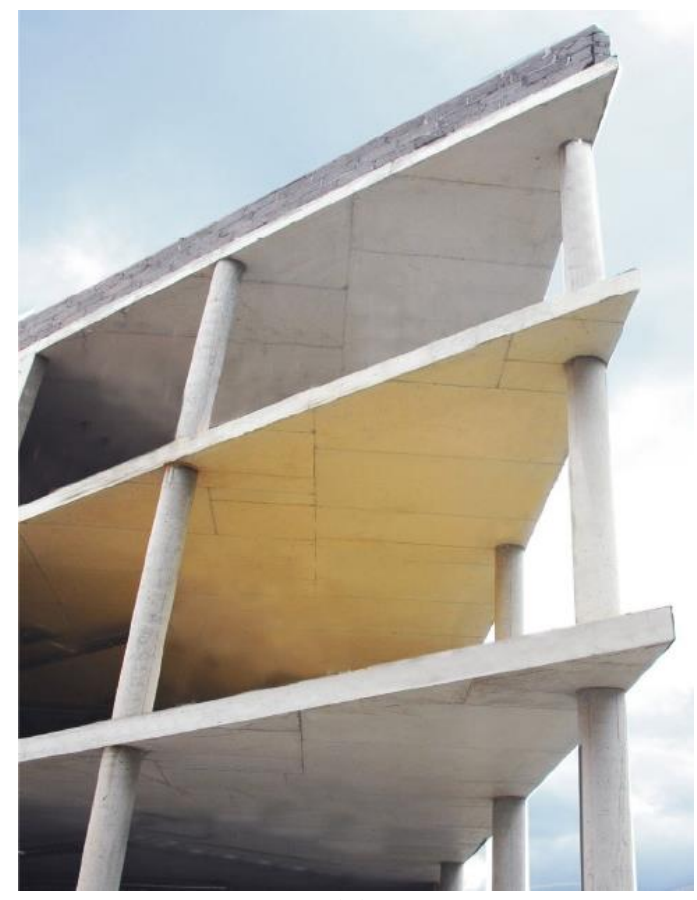

(a)

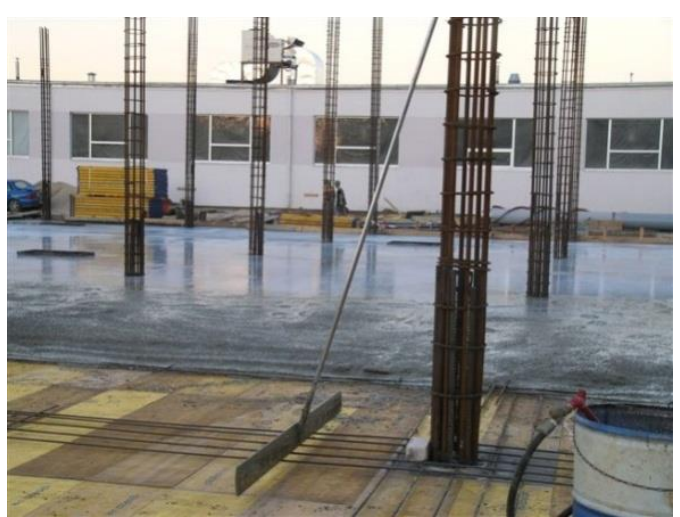

(b)

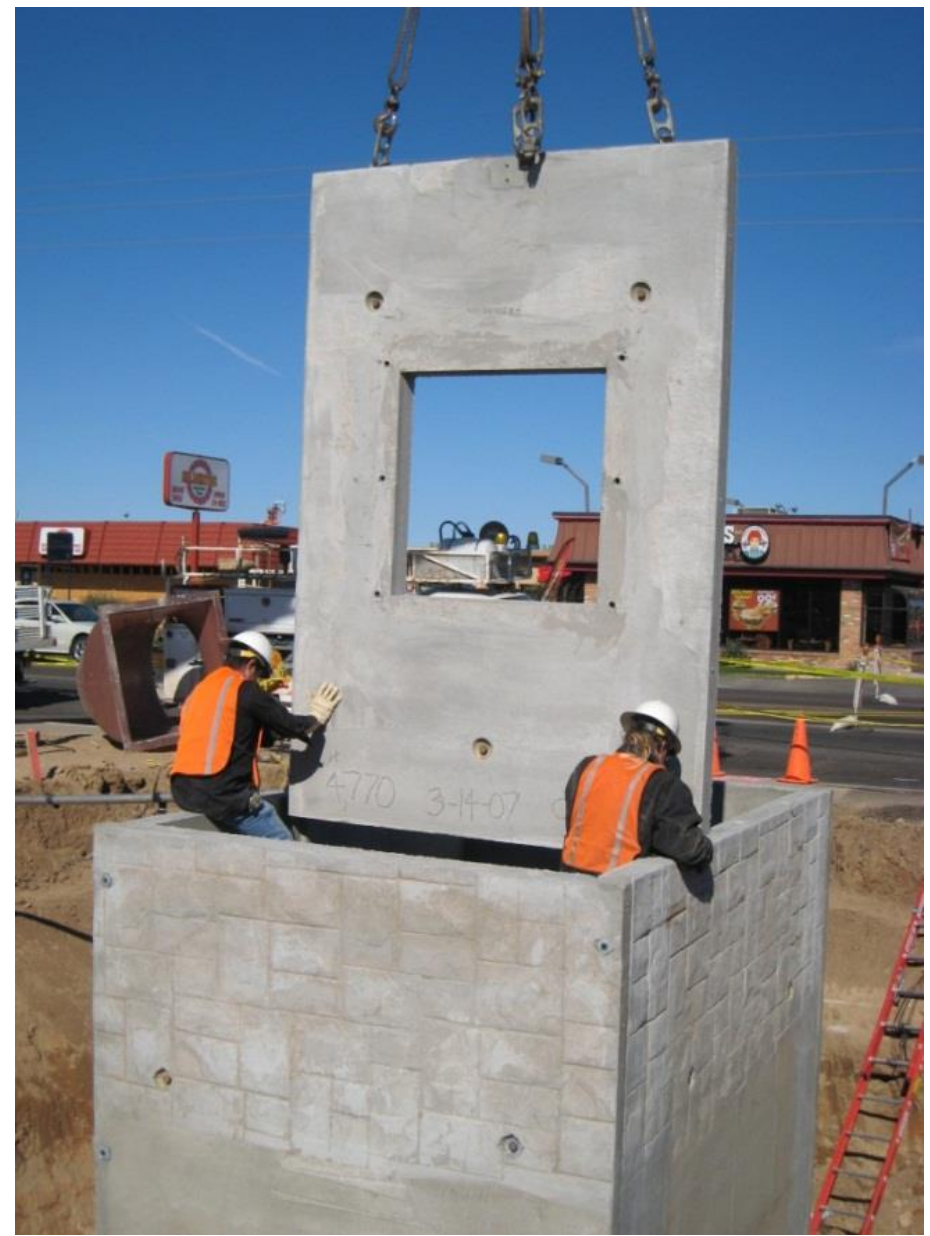

(c)

Fig. 1: Application of fiber reinforced concrete in: a) elevated slabs I, b) elevated slabs II, and c) water distribution infrastructure. 


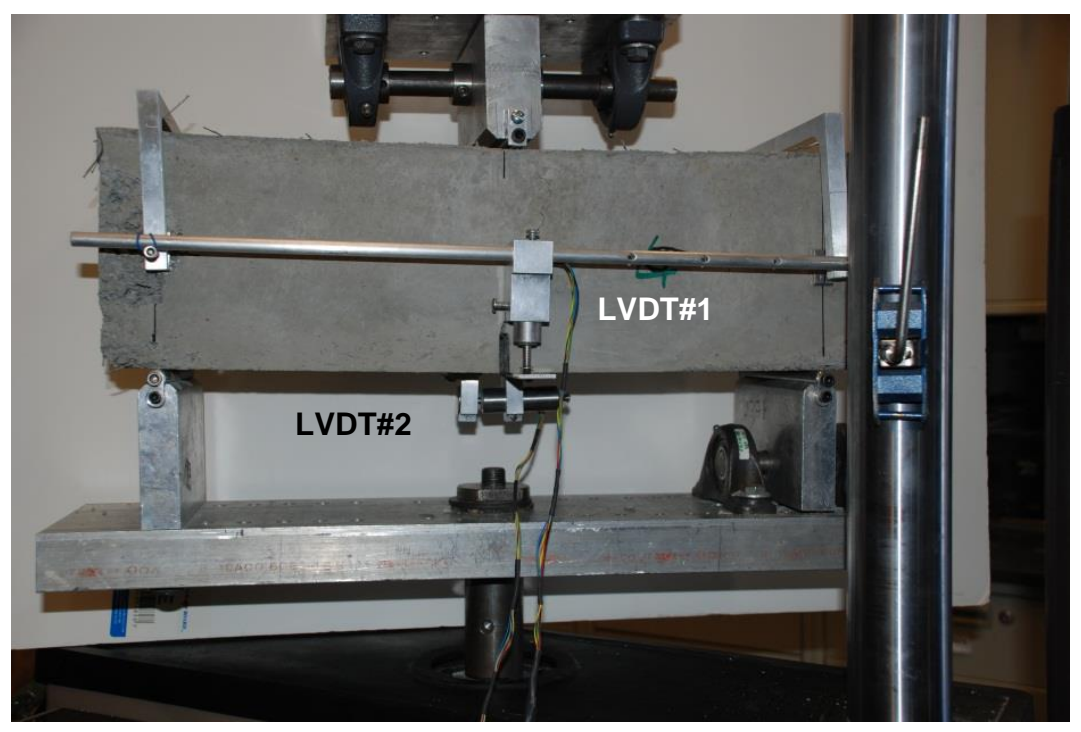

Fig. 2: Test setup for three-point bend notched flexural test. 


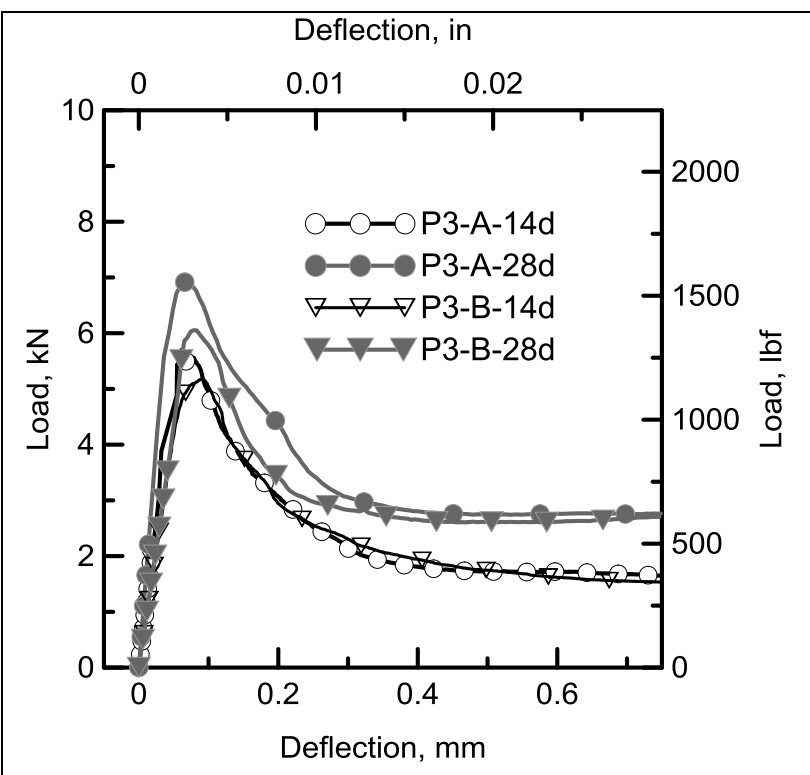

(a)

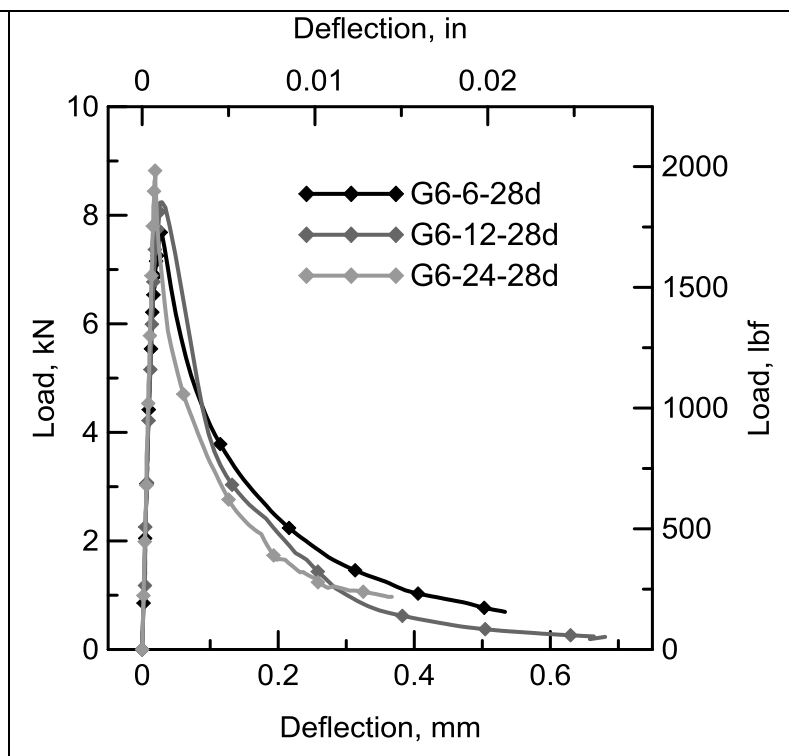

(b)

Fig. 3: a) Effect of curing time on load deflection response for polymeric fiber type A and B with fiber content of $3 \mathrm{~kg} / \mathrm{m}^{3}$ (Set 1), b) Effect of fiber type on load deflection response for glass with three different lengths at $6 \mathrm{~kg} / \mathrm{m}^{3}$ at age of 28 days (Set 2).

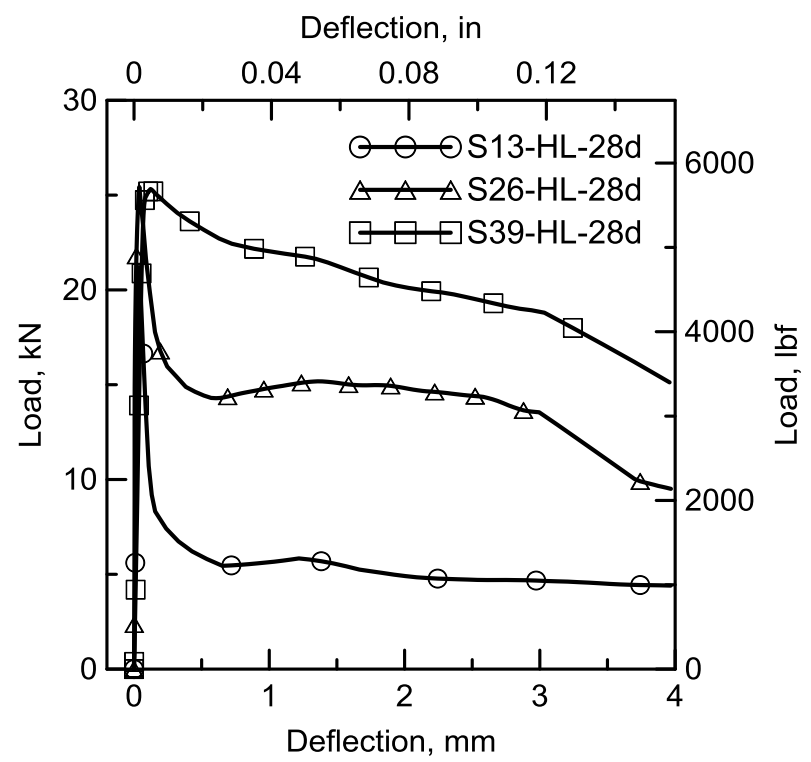

Fig. 4: Effect of fiber dosage on load deflection response for hooked steel fibers with large sample size $(150 \times 150 \times 450 \mathrm{~mm})$ at 28 days (Set 3$)$. 


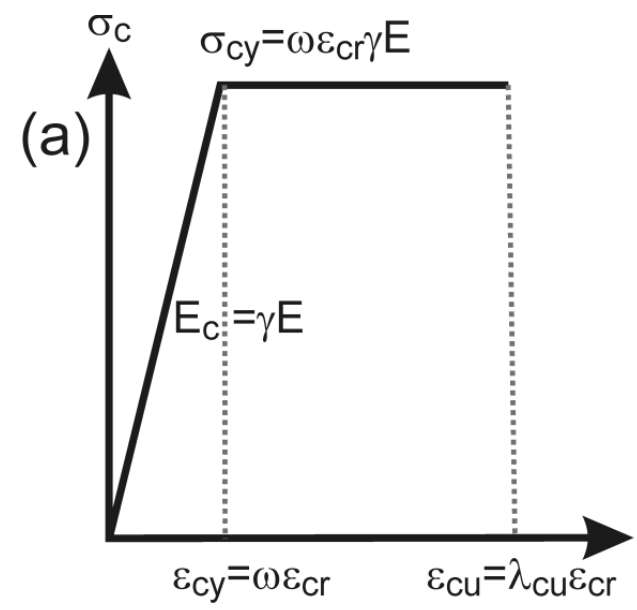

(a)

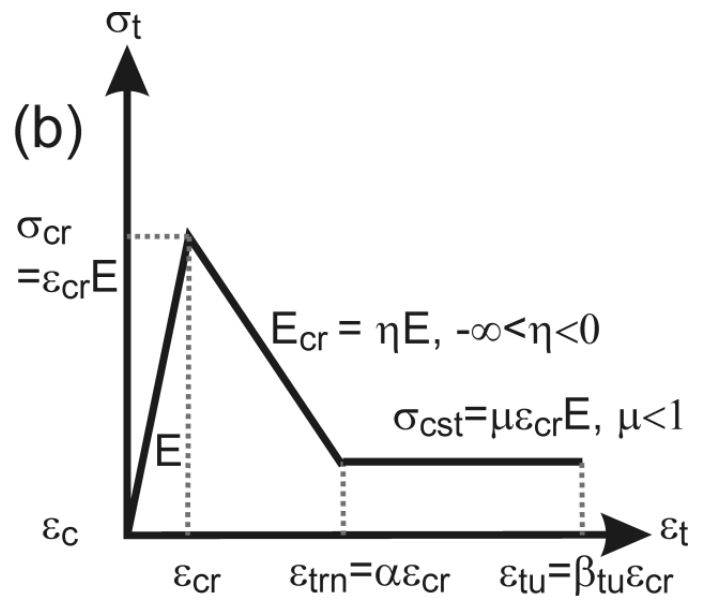

(b)

Fig. 5: Material models for FRC materials: (a) compression, (b) tension for strain softening composites

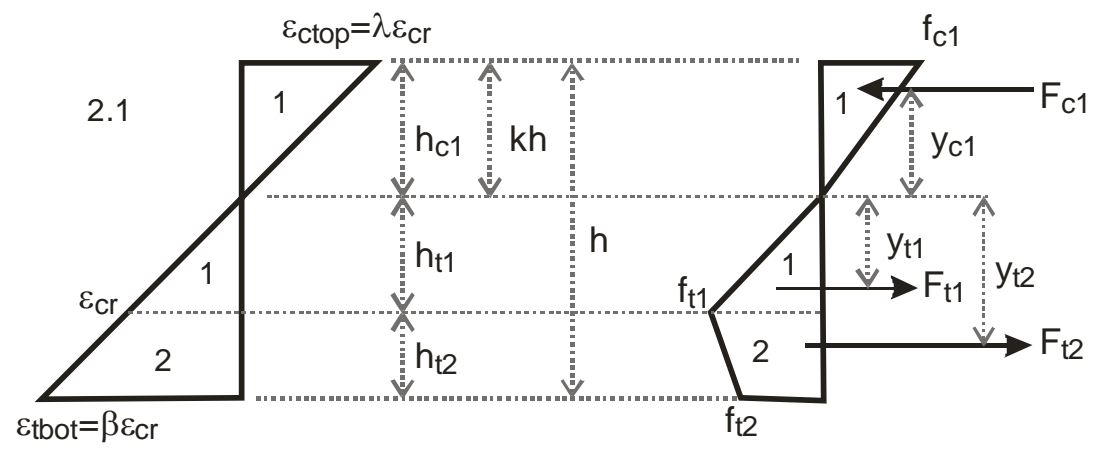

(a)

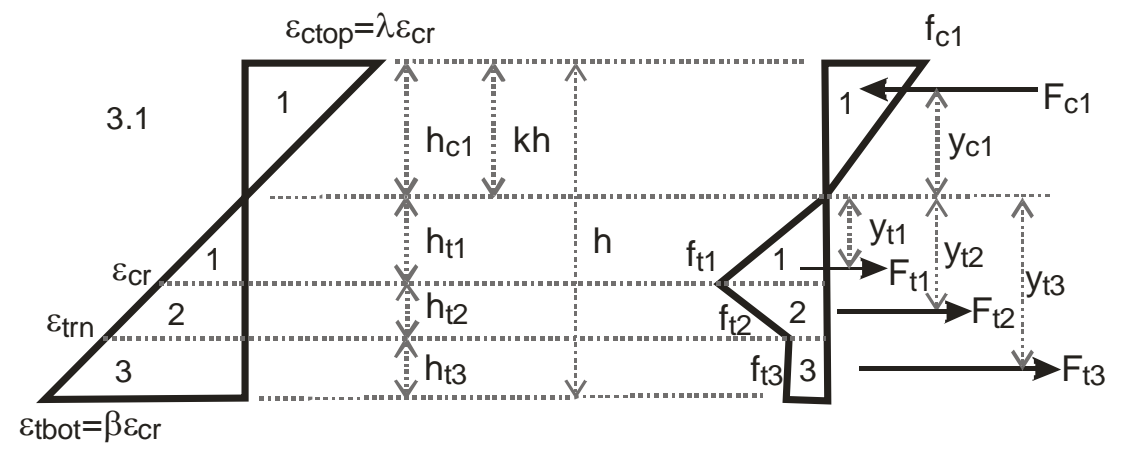

(b)

Fig. 6: Strain and stress diagrams at the post crack stage (Stages 2.1, and 3.1 Table 4), (a) strain distribution; and (b) stress distribution 


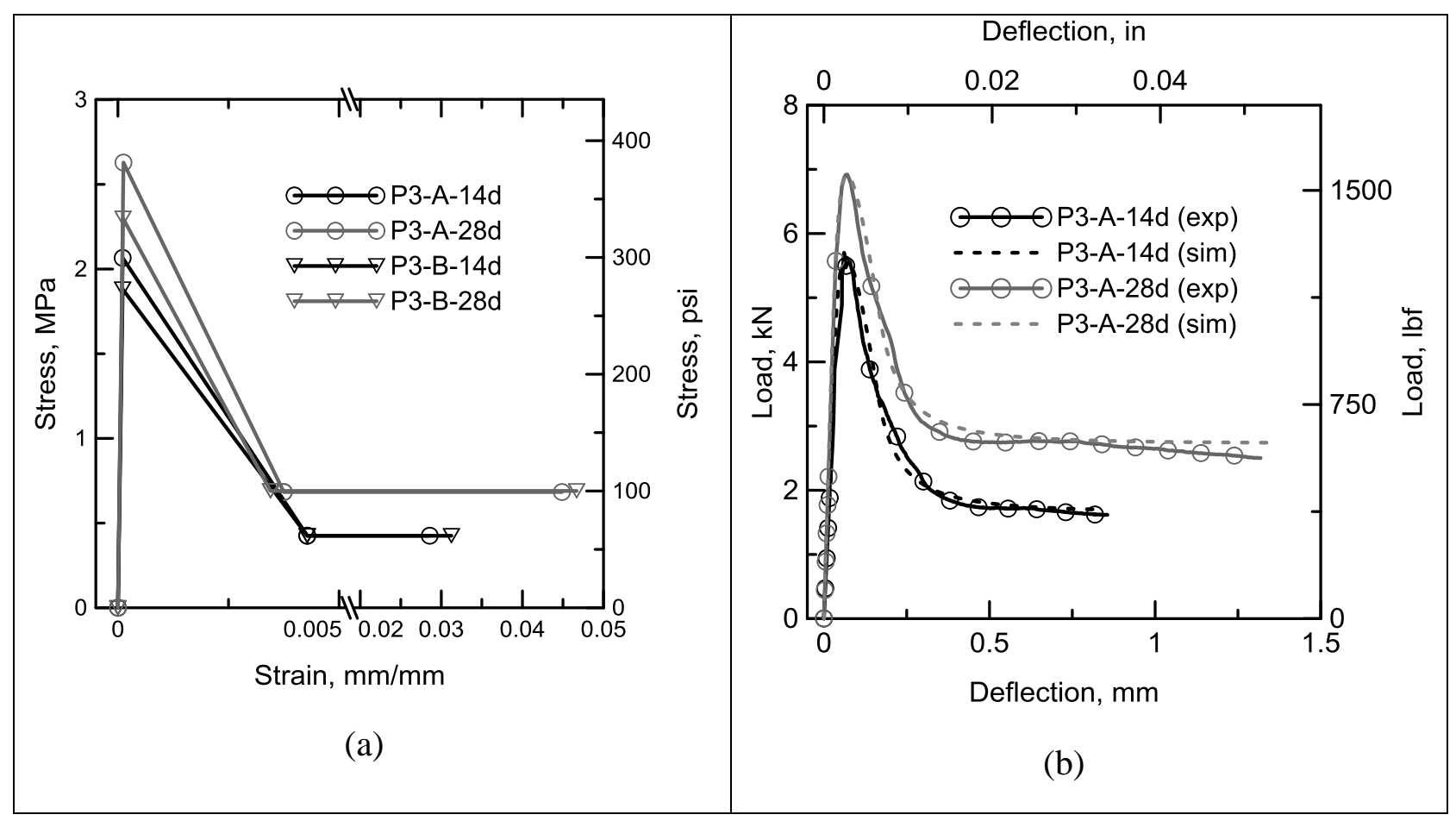

Fig. 7: a) Effect of curing time on back calculated tensile stress strain response, b) Effect of curing time on experimental and simulated load deflection response for polymeric fibers.(Set 1)

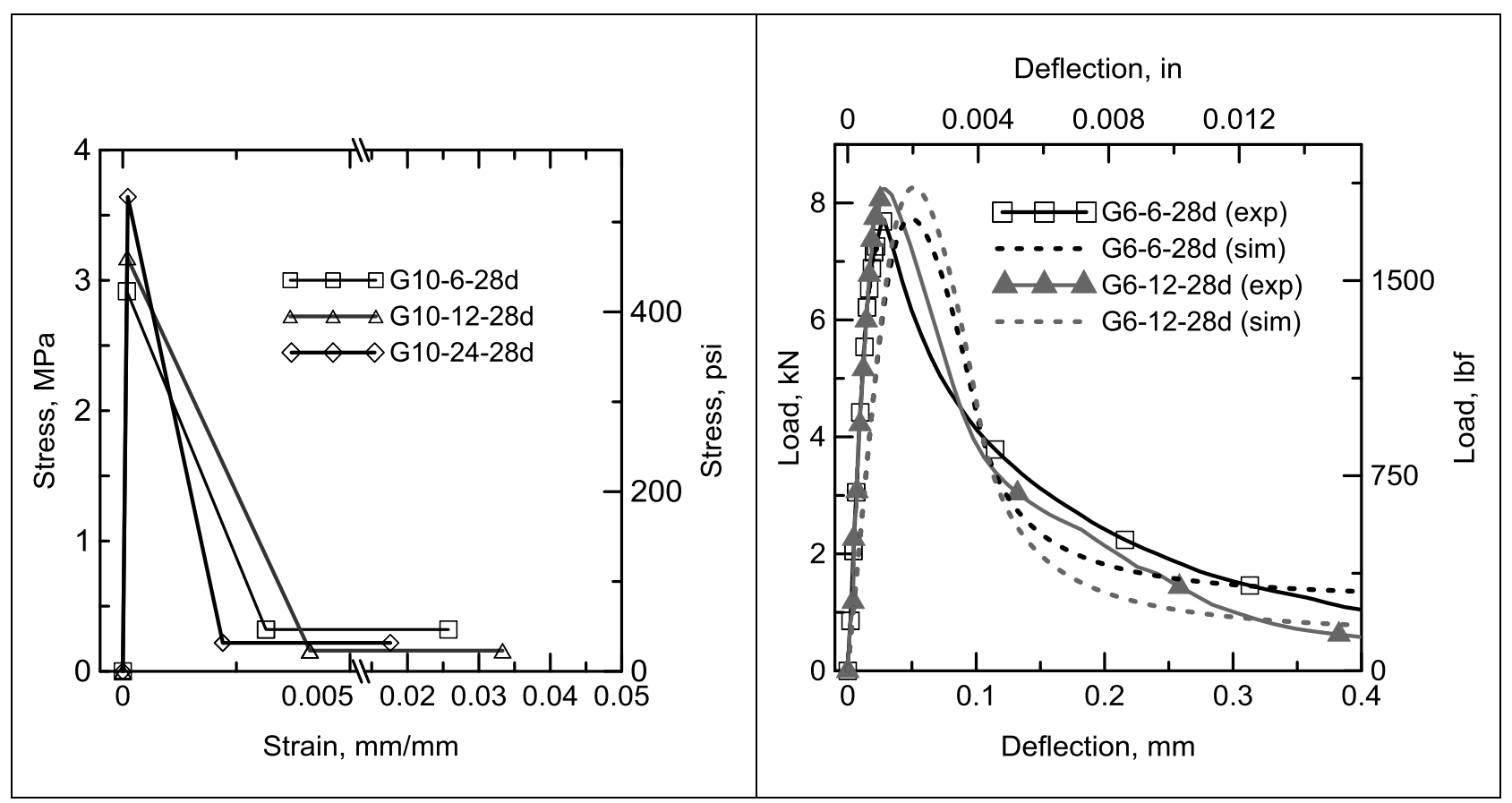

Fig. 8: a) Effect of curing time on back calculated tensile stress strain response, b) Effect of curing time on experimental and simulated load deflection response for glass fibers. (Set 2) 


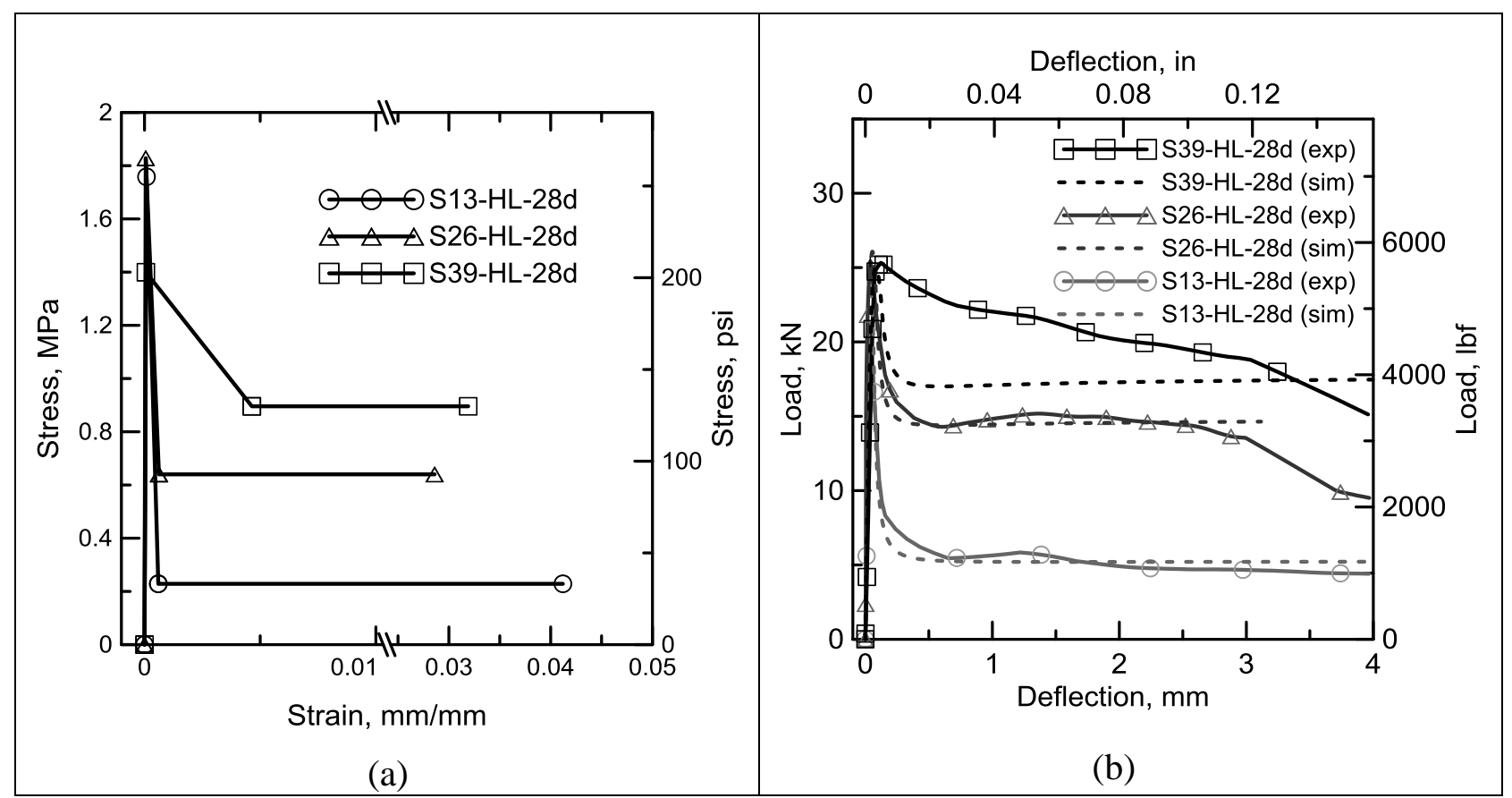

Fig. 9: a) Effect of steel fiber dosage on back calculated stress strain response, b) Effect of steel fiber dosage on experimental and simulated load deflection response
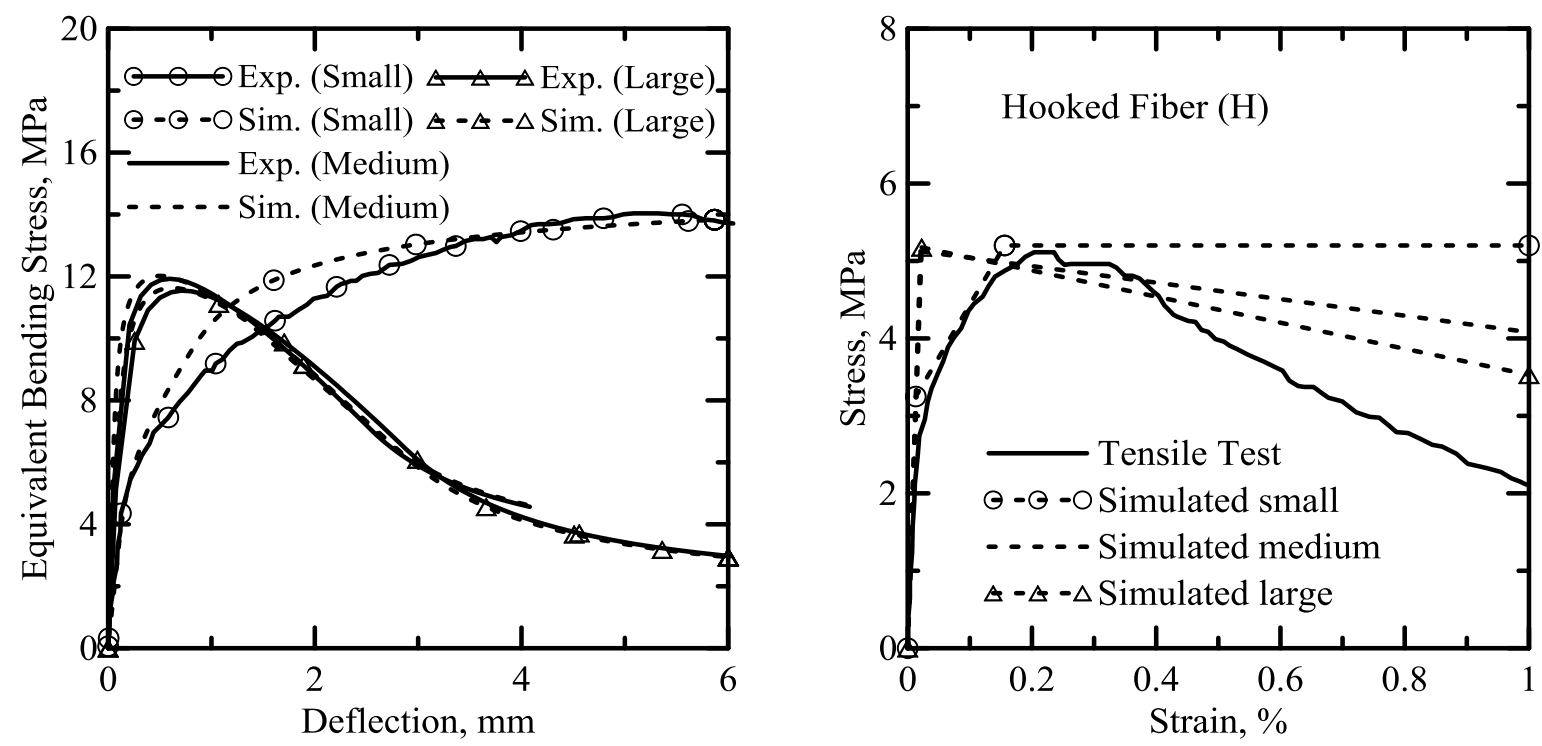

Fig. 10: a) Simulation of flexural responses of HPFRC materials with hooked-end fibers, b) comparing back calculated tensile stress-strain responses with experimental tensile stress-strain response for data set of Kim et al. [41]. (Set 4) 


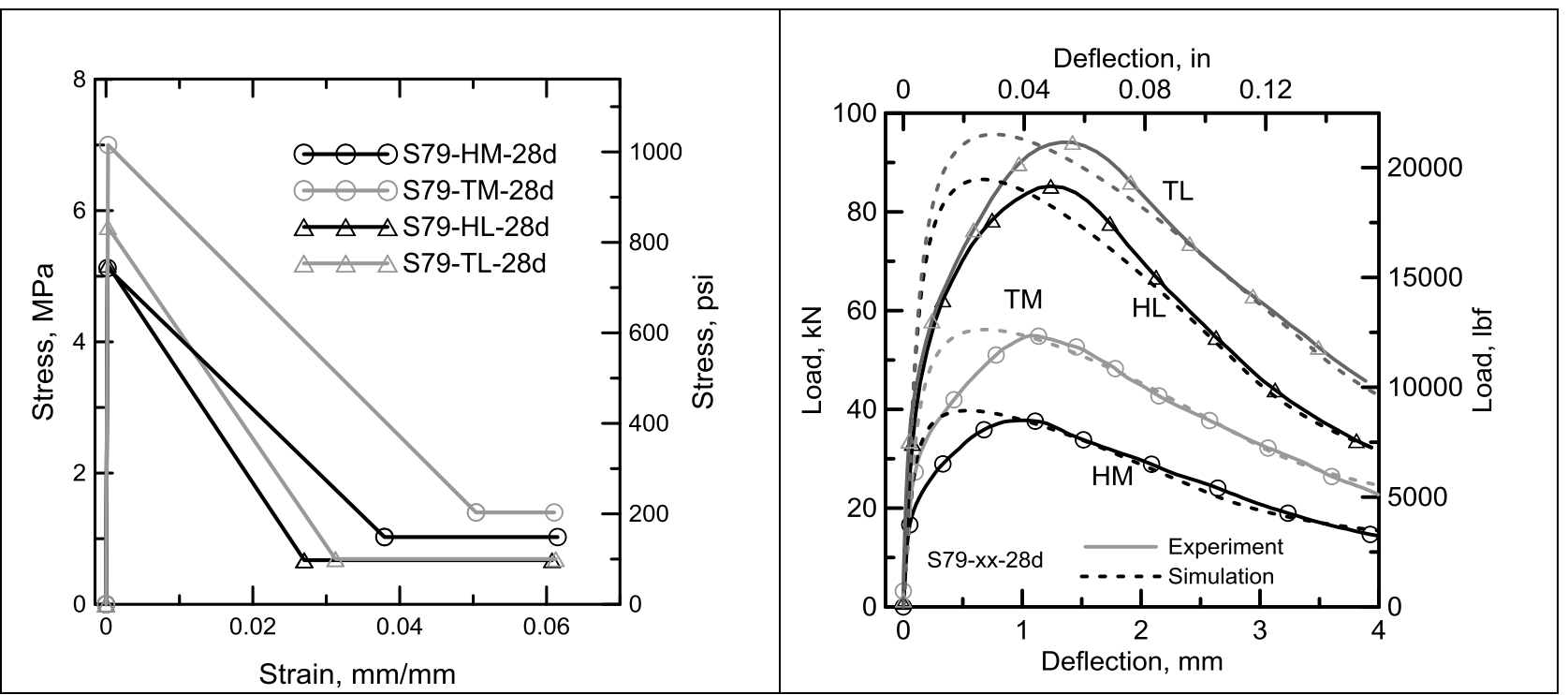

Fig. 11: a) Effect of sample size and steel fiber deformation on back calculated stress strain response ( $\mathrm{H}$ or $\mathrm{T}$ represents hooked or twisted and $\mathrm{M}$ or $\mathrm{L}$ represents Medium or Large), b) Effect of sample size and steel fiber content on experimental and simulated load deflection response 


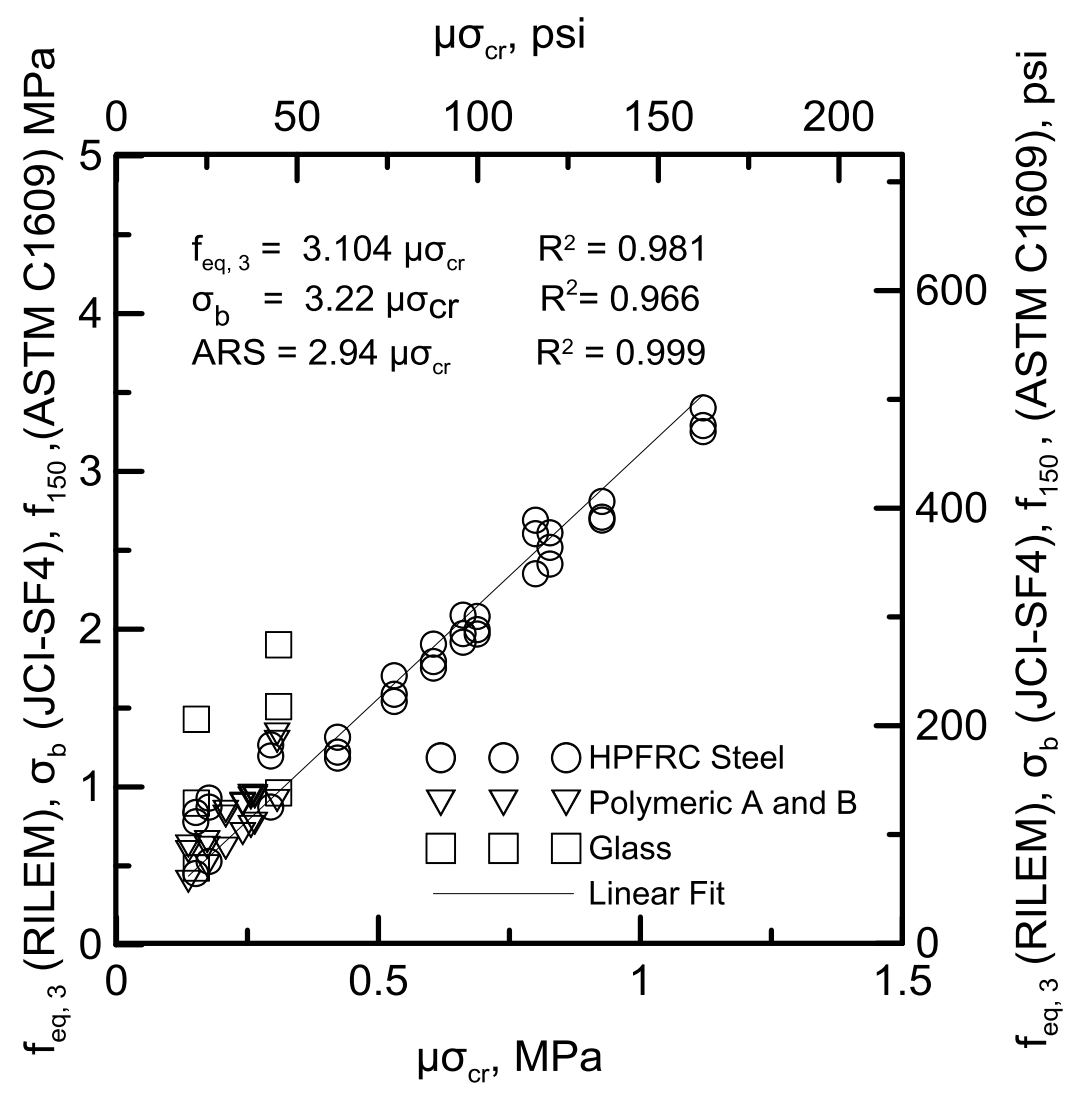

Fig. 12: Comparison of residual strength $\left(\mu \sigma_{\mathrm{cr}}\right)$ with JCI-SF4 residual parameter and RILEM residual parameterASTM-1609 residual parameter (Sets 1,2, 3, 4) 


\section{References}

1 diPrisco M, Plizzari G, Vandewalle L. Fibre reinforced concrete: new design perspectives. Mater Struct 2009;42(9):1261-81.

2 Walraven J. High performance fiber reinforced concrete: progress in knowledge and design codes. Mater Struct 2009;42(9):1247-60.

3 AASHTO-AGC-ARTBA (2001). "The use of state-of-the-practice of fiber reinforced concrete." Task Force 36 Report, Subcommittee on New Highway Materials, AASHTOAGC-ARTBA Joint Committee.

4 ACI (1997). "State-of-the-art report on fiber reinforced concrete." ACI 544.1R-96, American Concrete Institute, Farmington Hills, MI.

5 The Concrete Society (1994)."Concrete Industrial Ground Floors - A Guide to Their Design andConstruction.” Slough, Technical Report 34, 2nd Edition.

6 Mindess, S., Young, J.F., and Darwin, D. (2003), Concrete, Second Edition, PrenticeHall, Upper SaddleRiver, NJ.

7 Bentur, A. and Mindess, S. (1990). "Fibre Reinforced Cementitious Composites." Elsevier Applied Science, London.

8 Belletti B, Cerioni R, Meda A, Plizzari G. Design aspects on steel fiber-reinforced concrete pavements. J Mater Civ Eng 2008;20(9):599-607.

9 Sorelli LG, Meda A, Plizzari GA. Steel fiber concrete slabs on ground: a structural matter. ACI Struct J 2006;103(4):551-8.

10 Pelisser F, Santos Neto AB, La Rovere HL, Pinto RCA. Effect of the addition of synthetic fibers to concrete thin slabson plastic shrinkage cracking. Construction and Building Materials 24 (2010) 2171-2176.

11 Vondran, G.L. (1991). “Applications of Steel Fiber Reinforced Concrete.” Concrete International, 13(11),44-49.

12 Rollings, R. S. (1981). "Corps of Engineers Design Procedures for Rigid AirfieldPavements."Proceedings, Second International Conference on Concrete Pavement Design.Purdue University, WestLafayette, IN.

13 Parker, F. (1974).“'Steel fibrous concrete for airport pavement applications.'Technical Report 5-74-12,US Army Engineer Waterways Experiment Station, Vicksburg, MS.

14 Romualdi, J.P. and Batson, G.B. (1963)."Mechanics of Crack Arrest in Concrete." Journal EngineeringMechanics Division, ASCE, 89, 147-68.

15 Beckett, D., Van De Woestyne, T, and Callens, S. (1999). "Corner and edge loading on ground floors reinforced with steel fibers." Concrete, 33(3), 22-24. 
16 Falkner, H. and Teutsch, M. (1993). "Comparative investigations of plain and steel fibre reinforced industrial ground slabs." Institut Fur Baustoffe, Massivbau und Brandschutz, Technical University of Brunswick, Germany, No. 102.

17 Tatnall, P.C. and Kuitenbrouwer, L. (1992)."Steel fiber reinforced concrete in industrial floors", Concrete International, 14(12), 43-47.

18 Beckett, D. (1990). "Comparative tests on plain, fabric reinforced and steel fibre reinforced concrete ground slabs." Concrete, 24(3), 43-45.

19 Kukreja, C.B. et al. (1987). "Ultimate strength of fiber reinforced concrete slabs.'Proceedings of International Symposium on Fiber Reinforced Concrete, Madras, 1, 237-55.

20 Sham, S.H.R. and Burgoyne, C.J. (1986). "Load tests on Dramix steel fibre reinforced concrete slabs -A report to Sir Frederick Snow and Partners, Consulting Engineers" Imperial College of Science andTechnology, Department of Civil Engineering, Concrete Laboratories.

21 Ferrara L, Meda A. Relationships between fibre distribution, workability and the mechanical properties of SFRC applied to precast roof elements. Mater Struct 2006;39(288):411-20.

22 Gettu R, Barragán B, Garcia T, Ortiz J, Justa R. Fiber concrete tunnel lining. Concr Int 2006;28(8):63-9.

23 Bernard E. Correlations in the behaviour of fibre reinforced shotcrete beam and panel specimens. Mater Struct 2002;35(3):156-64.

24 Malhotra VM, Carette AGG, Bilodeau A. Mechanical Properties and durability of polypropylene fiber reinforced high-volume fly ash concrete for shotcrete applications. ACI Materials Journal, 91(5);478-486.

25 Armelin HS, Helene P. Physical and Mechanical Properties of Steel-Fiber Reinforced Dry-Mix Shotcrete. ACI Materials Journal. 92(3);258-267.

26 Morgan DR. Steel fiber reinforced shotcrete for support of underground openings in Canada. Concrete International, 13(11): 56-64.

27 Banthia N, Trottier JF, Wood D, Beaupre D. Influence of fiber geometry in steel fiber reinforced dry-mix shotcrete. Concrete International, 14(5):24-28.

28 American Concrete Institute. State of the art report on fiber reinforced shotcrete, 506.1R98(1998). AC1 Committee 506, American Concrete Institute, Detroit, MI.

29 Mobasher B. Mechanics of fiber and textile reinforced cement composites. CRC Press; 1st ed. (2011), Boca Raton, FL.

30 Glassfibre Reinforced Concrete: practical design and structural analysis / Publisher Beton-Verlag,Düsseldorf : 1995. 
31 Sukontasukkul P, Pomchiengpin W, Songpiriyakij S. Post-crack (or post-peak) flexural response and toughness of fiber reinforced concrete after exposure to high temperature. Construction and Building Materials 24 (2010) 1967-1974.

32 Sueki S, Soranakom C, Mobasher B, Peled A, Pullout-Slip Response of Fabrics Embedded in a Cement Paste Matrix, ASCE Journal of Materials in Civil Engineering, Vol. 19, No. 9, September 1, 2007.

33 Li C Y, Mobasher B. Finite element simulations of fiber pullout toughening in fiber reinforced cement based composites. AdvnCem Bas Mat 1998;7:123-132.

34 Roesler, J. and Gaedicke, C. (2004), "Fiber reinforced concrete for airfield rigid pavements," Technical Note 3 - Center of Excellence for Airport Technology (CEAT), Urbana, IL, October 2004, 11 pp.

35 Mobasher, B. and Li, C. Y., "Mechanical Properties of Hybrid Cement Based Composites," ACI Materials Journal, Vol. 93, No.3, pp.284-293, 1996.

36 BurattiN., Mazzotti C., Savoia M. Post-cracking behaviour of steel and macro-synthetic fibre-reinforced concretes. Construction and Building Materials 25 (2011) 2713-2722.

37 Soulioti DV, Barkoula NM, Paipetis A, Matikas TE. Effects of fibregeometry and volume fractionon the flexural behaviour of steel-fibre reinforced concrete. Strain (2011) 47, e535-e541.

38 ASTM C1609-10, "Standard Test Method for Flexural Performance of Fiber-Reinforced Concrete (Using Beam With Third-Point Loading)", ASTM Standard Book, V. 04.02, 2010.

39 RILEM TC 162-TDF, RILEM final recommendations on test and design methods for steel fibre reinforced concrete: bending test, Materials and Structures, 2002;35:579-582.

40 Japanese Concrete Institute, JCI-SF4, Methods of tests for flexural strength and flexural toughness of fiber reinforced concrete (using beam with third-point loading). 1984: 4556.

41 Kim D-J, Naaman AE, and El-Tawil S, Correlation between Tensile and Bending Behavior of FRC Composites with Scale Effect, Proceedings of FraMCoS-7, 7th International Conference on Fracture Mechanics of Concrete and Concrete Structures, May 23-28, 2010, Jeju Island, South Korea.

42 Soranakom C, Mobasher B. Closed form solutions for flexural response of fiber reinforced concrete beams. Journal of Engineering Mechanics 2007;133(8):933-41.

43 Soranakom C, Mobasher B. Closed-form moment-curvature expressions for homogenized fiber-reinforced concrete. ACI Material Journal 2007; 104(4):351-9.

44 Soranakom C, Mobasher B, Bansal S. Effect of material non-linearity on the flexural response of fiber reinforced concrete. Proceeding of the Eighth International Symposium on Brittle Matrix Composites BMC8, Warsaw, Poland, 2006:85-98. 
45 Soranakom C, Mobasher B. Correlation of tensile and flexural responses of strain softening and strain hardening cement composites. Cement \& Concrete Composites Vol. 30, 2008, pp. 465-477.

46 Soranakom C, Mobasher B. Flexural Analysis and Design of Textile Reinforced Concrete. 4th Colloquium on Textile Reinforced Structures (CTRS4), Dresden, Germany, June 3-5 2009, pp. 273-288.

47 Bakhshi M, Barsby C, Mobasher B (2012) Back-calculation of tensile properties of strain softening and hardening cement composites. In: Parra-Montesinos GJ, Reinhardt HW, Naaman AE (Eds) High Performance Fiber Reinforced Cement Composites 6 (HPFRCC6), Ann Arbo, MI, 83-90.

48 Barros, J. A. O., Cunha, V. M. C. F., Ribero, A. F., and Antunes, J. A. B. Postcrackingbehaviour of steel fibre reinforced concrete, Materials and Structures, Vol. 37, 2004.

49 fib (2010). "Model Code 2010 - First complete draft, Vol. 1”, Bulletin 55, 220-231. 